\title{
A Survey of Harmony Search Algorithm
}

\author{
Osama Abdel-Raouf \\ Operations Research and Decision Support Dept. \\ Faculty of Computers and Information \\ Menoufia University \\ ${ }^{*}$ Corresponding author
}

\author{
Mohamed Abdel-Baset Metwally \\ OperationsResearch and Decision Support Dept. \\ Faculty of Computers \& informatics \\ Zagazig University
}

\begin{abstract}
Harmony Search (HS) is a meta-heuristic algorithm which bases its operation on the musical improvisation process. Recently, HS has become a popular algorithm in the evolutionary computation fielddue to its superiority to many other algorithms. As a consequence, in this paper, HS algorithm, its improvements and applications in many fields, such as operations research and computer science, are discussed and analyzed. The survey investigates the difference between HS algorithms as well as its applications. To add to this, several future improvements are suggested.
\end{abstract}

\section{Keywords}

Harmony search algorithm, meta-heuristics, optimization, evolutionary algorithms

\section{INTRODUCTION}

The HS algorithm was formerly developed by Geem et al. in 2001[1], and is based on natural musical performance processes that happen when a musician searches for a better state of harmony, such as that during jazz improvisation. Jazz improvisation seeks to find musically pleasing harmony (a perfect state) as determined by an aesthetic standard, just as the optimization process seeks to find a global solution (a perfect state) determined by an objective function. The pitch of each musical instrument determines the aesthetic quality, just as the objective function value is determined by the set of values assigned to each design variable [1].

HS algorithm proved to be very successful in a wide range of optimization problems, such as water distribution and games[2-6], and showed better performance in comparison with other traditional optimization techniques. The advantages of HS can be summarized as shown below [6]:

i. HS algorithm imposes fewer mathematical requirements and does not require initial value settings of the decision variables.

ii. As the HS algorithm uses stochastic random searches, derivative information is also needless.

iii. HS algorithm generates a new vector, after considering all of the existing vectors, while the genetic algorithm (GA) only considers the two parent vectors. These features increase the flexibility of the HS algorithm and produce better solutions.

iv. HS is good at identifying the high performance regions of the solution space at a reasonable time,

On the other hand, like any other Evolutionary algorithm(EA), the main con of HS is it may getstuck into local solutions in solving multimodal problems. However, many studies have been introduced to improve the performance of HS.This will be discussed in a latter section.

As a result of its significance in the EA field, in the paper, HS and its improvements are presented and analyzed. Furthermore, the real-world applications of HS are discussed and proposed future trends are suggested.

This paper is organized as follows, section 2 discusses HS algorithm. The improvements of HS are then presented in section 3, while section 4 shows the real-world application of HS. Section 5 shows conclusions and suggestions for future work.

\section{BASIC HARMONY ALGORITHM}

In HS algorithm, the harmony memory(HM) stores the feasible vectors, which are all in the feasible space. When a musician improvises one pitch, usually one of three rules is used:

i. Generating any one pitch from his/her memory, i.e. choosing any one value from harmony memory, defined as memory consideration;

ii. Generatinga nearby pitch of one pitch in his/her memory, i.e. choosing anadjacent value of one value from harmony memory, defined as pitch adjustments;

iii. Generating totally a random pitch from possible sound ranges, i.e. choosing totally random value from the possible value range, defined as randomization

Similarly, when each decision variable chooses one value in the HS algorithm, it can apply one of the abovementioned rules in the whole HS algorithm. If the new harmony vector is better than the worst harmony vector in the harmony memory, then the new harmony vector will changeit. This procedure is repeated until a stopping criterion is satisfied[12-14]. HS algorithm optimization procedure, shown below, consists of five steps.

\section{Step 1: Parameters Initialization}

The optimization problem which is specified as follows:

$$
\text { Minimize } f(x)
$$

subject to

$$
x_{j} \in X_{j}=1,2, \ldots, N,
$$

where $f(x)$ is an objective function; $x$ the set of each decision variable $x_{j} ; N$ the number of decision variables, $X_{j}$ the set of the possible range of values for each decision variable, that is 
$x_{j}{ }^{\min }$ and $x_{\mathrm{j}}^{\max }$ are the lower and upper boundaries of the $j_{\mathrm{th}}$ decision parameter, respectively.

HS algorithm parameters are also specified in this step, namely, the harmony memory size (HMS), or the number of solution vectors in the harmony memory, harmony memory considering rate (HMCR), pitch adjusting rate (PAR), bandwidth distance(BW), and the number of improvisations (NI), or stopping criterion, and the harmony memory (HM) where all the solution vectors are stored.

\section{Step 2: Harmony Memory Initialization and Evaluation}

A random initial population is generated,such as:

$$
x_{i, j}^{0}=x_{j}^{\text {min }}+r_{j}\left(x_{j}^{\text {max }}-x_{j}^{\text {min }}\right),
$$

where $i=1,2, \ldots H M S ; j=1,2, \ldots, N$ and $r_{j} \in[0,1]$ is a uniformly distributed random number generated new for each value of $j$. Solution vectors in HM are analyzed, and their objective function values are then calculated.

\section{Step 3: Improvisation}

In this step, a new harmony vector is generated based on three rules, namely, memory consideration, pitch adjustment and random selection. The value of a design variable can be selected from the values stored in HM with a probability HMCR. It can be further adjusted by moving to a neighbor value of a selected value from the HM with a probability of pitch adjusting rate (PAR), or, it can be selected randomly from the set of all candidate values without considering the stored values in HM, with the probability of (1 - HMCR).

\section{Step 4: Harmony Memory Update}

If the new harmony vector is better than the worst vector, based on the objective value and/or constraint violation, the new vector will replace the worst one.

\section{Step 5: Termination criterion check}

HS algorithm is terminated if the stopping criterion (e.g. maximum number of improvisations) has been met. Otherwise, steps 3 and 4 are repeated.

Algorithm 1 shows pseudo-code of HS algorithm, while figure 1shows the HS algorithm flowchart

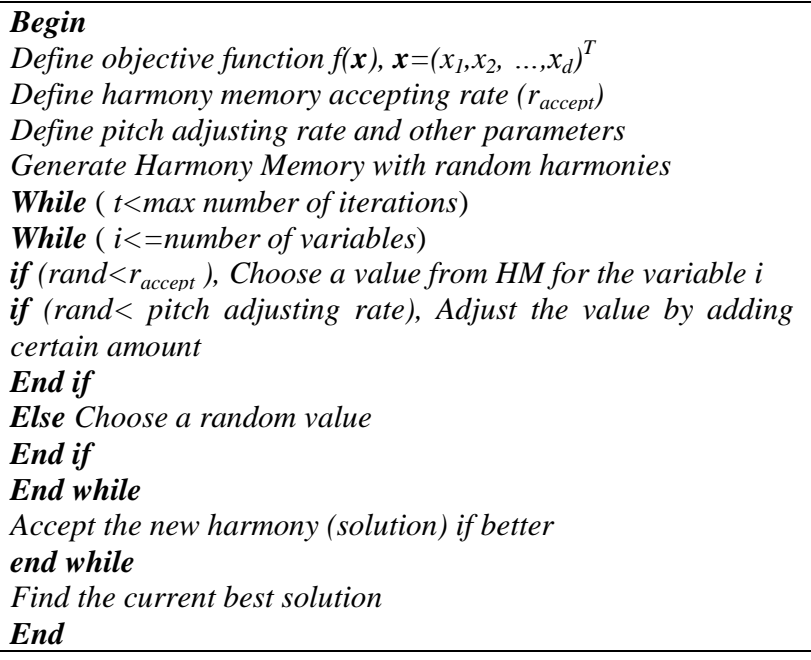

Algorithm 1. HS algorithm pseudo-code

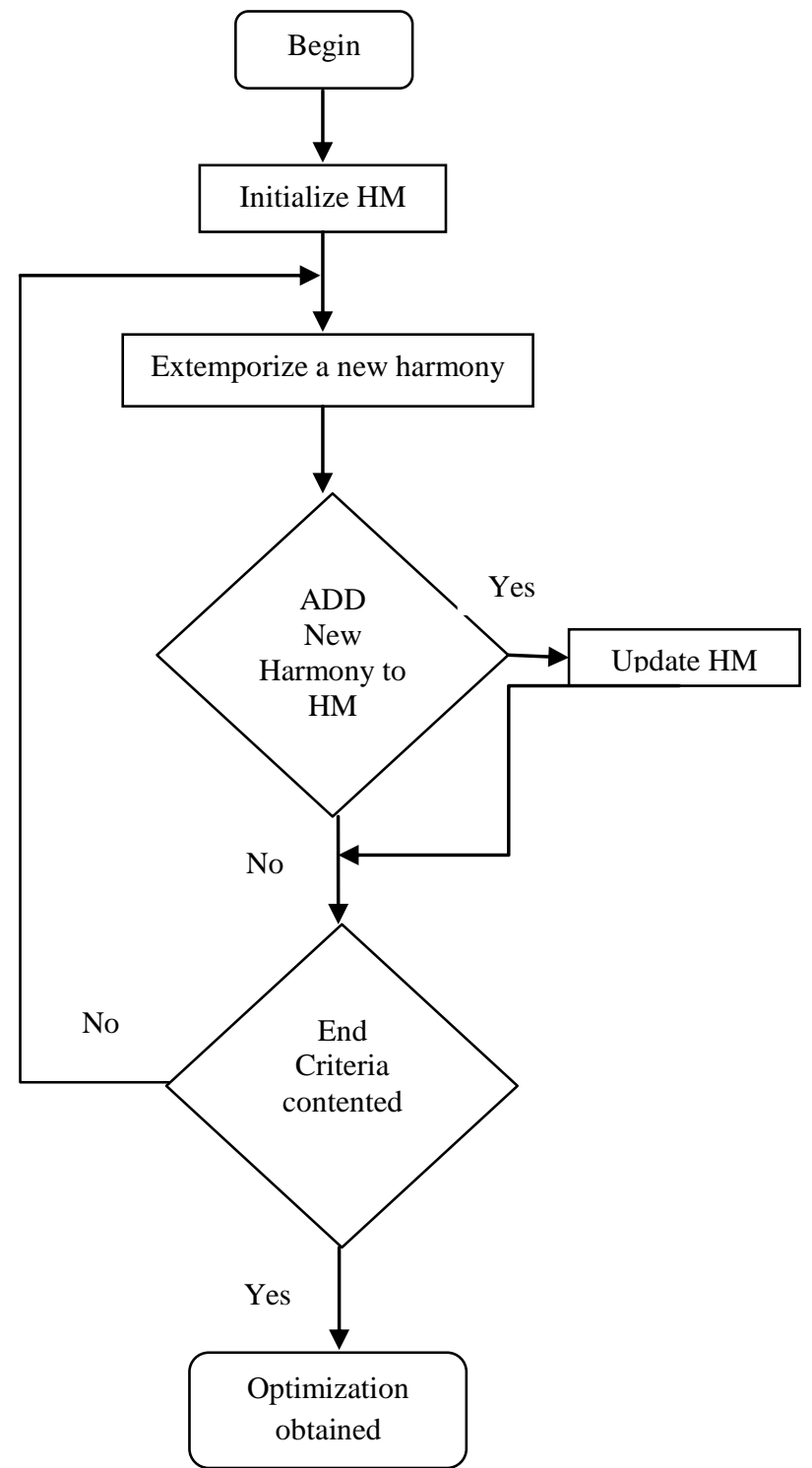

Fig. 1 Flowchart of HS

\subsection{HS over other optimization strategies}

Heuristic algorithms are normally set to find a good solution to an optimization problem by 'trial-and-error' in a logical amount of computing time. Here 'heuristic' means to 'find' or 'search' by trials and errors. There is no assurance to find the optimal solution, though it might find a better solution than a learned deduce one. Generally speaking,heuristic methods are local search methods, because their searches concentrate on the local variations, and the best solution can locate outside of this local region. Metaheuristic means to find the solution using higher-level techniques, though certain trial-and-error processes are still used. Metaheuristicsrefers to all modern higher-level algorithms, including Evolutionary Algorithms (EA)[15] including Genetic Algorithms (GA), Simulated Annealing (SA)[16], Tabu Search (TS)[17], Ant Colony Optimization (ACO)[18,19], Particle Swarm Optimization (PSO)[20], Bee colony Algorithms (BCA)[21;22], Firefly Algorithms (FA)[22,23], and Harmony Search [4].

There are two important components in metaheuristics: intensification and diversification. Diversification is often in the form of randomization with a random component attached 
to a deterministic component in order to explore the search space effectively and efficiently, while intensification is the exploitation of past solutions so as to select the potentially good solutions [4-6]. Any successful metaheuristic algorithm requires a good balance between these two important, seemingly opposite, components [6]. Another important feature of modern metaheuristics is that an algorithm is either trajectory-based or population-based. The optimal balance of diversification and intensification is necessary, and such a balance itself is an optimization process. Fine-tuning of parameters is often required to improve the efficiency of an algorithm for a particular problem. There is No Free Lunch in any optimization problem [21]. A substantial amount of studies might be required to choose the right algorithm for the right optimization problem [16], though a systematic guidance lacks for such a choice. In the HS algorithm, diversification is essentially controlled by the pitch adjustment and randomization-here there are two subcomponents fordiversification, which might be an important factor for the high efficiency of the HS method. The first subcomponent of playing a new pitch (or generating a new value) via randomization would be at least at the same level of efficiency as in other algorithms that handle randomization.

However, an additional subcomponent for HS diversification is the pitch adjustment operation performed with the probability of Pitch adjustment rate. Pitch adjustment is carried out by tuning the pitch within a given bandwidth. A small random amount is added to or subtracted from an existing pitch (or solution) stored in HM. Essentially, pitch adjustment is a refinement process of local solutions. Both memory consideration and pitch adjustment ensure that good local solutions are retained while the randomization makes the algorithm to explore global search space effectively. The subtlety is the fact that HS operates controlled diversification around good solutions, and intensification as well. The randomization explores the search space more widely and efficiently;while the pitch adjustment ensures that the newly generated solution is good enough,or not too far from existing good solutions. The intensification in the HS algorithm is represented by the harmony memory acceptingrate $r_{\text {accept }}$. A high harmony acceptance rate means that good solutions fromthe history/memory are more likely to be selected or inherited. This is equivalent to acertain degree of elitism. Obviously, if the acceptance rate is too low, solutions willconverge moreslowly. As mentioned earlier, this intensification is enhanced by thecontrolled pitch adjustment. Such interactions between various components could beanother important factor for the success of the HS algorithm over other algorithms, asit will be demonstrated again in other chapters of this book.In addition, the structure of the HS algorithm is relatively easier. This advantagemakes it very versatile to combine HS with other metaheuristic algorithms [22]. Foralgorithm parameters, there are some evidences to suggest that HS is less sensitive tochosen parameters, which means that we may not have to fine-tune these parametersto get quality solutions.Furthermore, the HS algorithm is a population-based metaheuristic, which meansthat a group of multiple harmonies can be used in parallel. Proper parallelism usuallyleads to better performance with higher efficiency. The good combination of parallelismwith elitism as well as a fine balance of intensification and diversification is thekey to the success of the HS algorithm [5,7,24-28].

\section{IMPROVEMENTS OF HS ALGORITHM}

Significant research studies have been proposed to improve HS's performance. Among the transformations, and hybridizations, HS improvements include the following: Improved harmony search(IHS), the global-best harmony search (GHS, Global-best Harmony Search) [7]) and the Novel global harmony search (NGHS) [9]. These new algorithms have been considered for development of various existing optimization techniques

\subsection{Improved Harmony Search (IHS)}

Improved Harmony Search is a new harmony algorithm proposed in 2007 by Mahdavi et al. [8]. It applies a method for generating new solution vectors based on the dynamic adjustment of the PAR (pitch adjustment rate) and $b w$ (pitch adjustment bandwidth) parameters, thus doing improved accuracy and convergence speed. In this deviation only the step that creates a new harmony is adjusted. PAR and $b w$ change dynamically with the number of generations and are calculated using the following formulas:

$$
P A R=P A R_{\text {min }}+\frac{\left(P A R_{\max }-P A R_{\min }\right)}{N I-1} \times(c i-1)(3)
$$

where PAR is the Pitch Adjustment Rate for each improvisation (iteration), $\mathrm{PAR}_{\min }$ minimum pitch adjustment rate, $\mathrm{PAR}_{\max }$ maximum pitch adjustment rate, NI total number of improvisations (Maximum iteration) and $\mathrm{Ci}$ current iteration.

$$
B W=B W_{\text {max }} e^{\left(\frac{\ln \left(\frac{B W_{\min }}{\left.B W_{\max }\right)}\right.}{N I} \times(c i-1)\right)}(4)
$$

where $B W$ is bandwidth for each iteration, $\mathrm{BW}_{\min }$ minimum bandwidth, $\mathrm{BW}_{\max }$ maximum bandwidth

The PAR parameter increases linearly with the number of generations (some papers declare otherwise with numerical simulation results [29]), while $b w$ decreases exponentially (for better $b w$, Das et al. [30] provided a theoretical background of the exploratory power of HS). Given this change in the parameters, IHS improves the performance of HS, since it finds better solutions. However, a major drawback of the IHS is that the user needs to specify the values for $b w_{\min }$ and $b w_{\max }$ which are difficult to deduce and problem dependent [7].

In [11], a more PAR function using the idea of simulated annealing has been proposed which increases the robustness of algorithm and therefore leads to a highly reliable algorithm. in [12], some improvements on convergence of HS have also been performed.

\subsection{Global-best Harmony Search $\operatorname{Algorithm(GHS)~}$}

Another improvement is called global-best harmony search (GHS).It is stimulated by the concept of particle swarm optimization (PSO) [7]. It modifies the pitch adjustment step of the HS such that the new harmony vector can imitate the best harmony in the harmony memory. Therefore, this approach replaces the $B W$ parameter and adds a social dimension to the HS [7]. GHS has exactly the same steps as the IHS with the exception that New Harmony Improvisation step as illustrated in below: 
for $j=1 \ldots N(N=$ number of decision variables $)$

do

$R l=U(0,1)$

If $(r 1<$ memory consideration $)$

$X[i, j]$ will be randomly chosen from harmony memory

$R 2=U(0.1)$

If $(r 2<$ pitch adjustment)

best $=$ index of the best harmony in the harmony memory

$M \in(1, \ldots, N)$

$x[i, j]=x[$ best,$M]$

end if

else (random selection)

$x[i, j]=x \in$ value set

end if

end do

Generally speaking, GHS was found better than IHS and HS when applied to problems with high dimensions and noise[7], for example in the water network design, GHS was better than HS in small $(n=8)$ and medium $(n=34)$ sized problems, GHS was worse than HS in large $(n=454)$ sized problems[31]

\subsection{A Novel Global Harmony Search algorithm (NGHS)}

NGHS proposes a hybridization between the harmony search swarm based optimization and mutation gene [9]. In NGHS, the improvisation step of the HS is modified such that the new harmony can take off the global best harmony in the HS. Overall, NGHS is different than HS in three aspects:

i. HMCRand PARare excluded from the NGHS, and genetic mutation probability (pm) is included.

ii. The NGHS modifies the improvisation step of the HS, and it works as follows:"best" and "worst" are the indexes of the global best harmony and the worst harmony in $\mathrm{HM}$, respectively. $r 1_{i}, r 2_{i}, r 3_{i}$ are all uniformly generated random numbers in $[0,1] . r l_{i}$ is used for position updating, $r 2_{i}$ determines whether the NGHS should carry out "genetic mutation and " $r 3_{i}$ is used for " genetic mutation".

iii. After improvisation, the NGHS replaces the worst harmony $x^{\text {worst }}$ in HM with the new harmony $x^{\prime}$ even if $x^{\prime}$ is worse than $x^{\text {worst }}[9]$.

NGHS steps are shown below:

for each $i \in[1, N] d o$

$x_{R}=2 \times x_{i}^{\text {best }}-x_{i}^{\text {wost }}$

if $x_{R}>x_{i U}$

$x_{R}=X_{i U}$

elseif $x_{R}<x_{i L}$

$x_{R}=x_{i L}$

end

\author{
$x_{i}^{\prime}<x_{i}^{\text {worst }}+r 1_{i} \times\left(x_{R}-x_{i}^{\text {worst }}\right) \quad / /$ position updating \\ if $r 2_{i} \leq p_{m}$ then \\ $x_{i}^{\prime}<x_{i L}+r 3_{i} \times\left(x_{i U}-x_{i L}\right) \quad / / g$ enetic mutation \\ end \\ end
}

\subsection{Chaotic harmony search algorithms}

In recent years, theories and applications of nonlinear dynamics, especially of chaos, have drawn more and more attention in many fields. One is chaos controlling, and synchronization. Another field is the potential applications of chaos in various disciplines including optimization.Chaos is a deterministic, random-like processfound in nonlinear, dynamical system, which isnon-period, non-converging and bounded.Moreover, it has a very sensitive dependenceupon its initial condition and parameter. Thenature of chaos is apparently random andunpredictable and it also possesses an elementof regularity. Mathematically, chaos israndomness of a simple deterministic dynamicalsystem and chaotic system may be considered assources of randomness [79]. A chaoticmap is a discrete-time dynamical system $\left(x_{k+1}=f\left(x_{k}\right)\right.$, $\left.0<x_{k}<1, k=0,1,2, \ldots\right)$ running in the chaotic state. The chaotic sequence $\left\{x_{k}: k=0,1,2, \ldots\right\}$ can be used as spreadspectrum sequence and as a random number sequence. The use of chaotic sequences in HS can be helpful to improve the global convergence, and to allow escaping from local solutions than the classical HS algorithm which uses fixed values for HMCR, PAR and bw. In the chaotic HS algorithm, when a random number is needed by the classical HS algorithm, it is generated by iterating one step of the chosen chaotic map that has been started from a random initial condition at the first iteration of the HS [79].

Chaotic harmony search (CHS) algorithms may be simply classified and described as follows:

i. Initial HM is generated by iterating the selected chaotic maps until reaching to the HMS.

ii. In it, PAR value is not fixed. However, itis modified by the selected chaotic maps as follows:

$\operatorname{PAR}(t+1)=f(\operatorname{PAR}(t)), 0<\operatorname{PAR}(t)<1, t=0,1,2, \ldots$

iii. Here, $B W$ is modified by the selected chaotic maps as follows:

$B W(t+1)=f(B W(t)), 0<B W(t)<1, t=0,1,2, \ldots$

iv. Both PAR and $B W$ values are modified by the selected chaotic maps as follows

$$
\begin{aligned}
& \operatorname{PAR}(t+1)=f(\operatorname{PAR}(t)), \quad 0<\operatorname{PAR}(t)<1, \quad t=0,1,2, \ldots \\
& B W(t+1)=f(B W(t)), \quad 0<B W(t)<1, \quad t=0,1,2, \ldots
\end{aligned}
$$

$\mathrm{v}$. $\mathrm{i}$ and ii are combined, that is initial HM is generated by iterating the selected chaotic maps and PAR value has been modified by the selected chaotic maps when needed.

vi. $i$ and ii are combined, that is initial HM is generated by iterating the selected chaotic maps and $B W$ value has been modified by the selected chaotic maps when needed.

vii. In this approach i, ii, and iii are combined.

\section{HS APPLICATIONS}

HS has been considered by many authors as a very successful algorithm, competing with other meta-heuristics, such as PSO, 
Tabu Search (TS) and GA. In recent years, several investigations have been developed around HS, and been applied to various optimization problems in computer science, operations research,science and engineering, among which are included: daily applications, computer science, electrical engineering[40-46], civil engineering[47-55], mechanical engineering[56-59], biomedical[60-62] ,economics[63], transport $[28,64]$, ecology[51,65,66], among others [14, 32].we mention the important applications related to operations research(OR) and Computer science (CS).

\subsection{OR Applications}

When it comes to everyday problems, reference the application of optimization algorithms in solving common problems in human development and for which there is no science or research field right that identify. Some of these problems are:

\section{Transport-Related Problems}

A Travelling Sales Man (TSP) problem with 20-city was solved in $[28,64]$ and with 51 -city TSP was also presented in [1], both using a modified HS algorithm. A school bus routing problem was investigated in [86] and briefly in [32]. The problem was to find the required number of school buses and the best route for each bus subject to constraints on the bus seating capacity and maximum allowable journey time. A generalized orienteering problem, for the best touring in China, was solved using a modified HS method in [84]. The aim was to find the tour route that maximized the collective tourism opportunities offered by eachcity, subject to a constraint on the maximum tour length. A parameter estimation problem for the annual energy demand of the Turkish transportation sector was reported in [85].

\section{Multi-objective optimization problems}

Geem and Hwangbo [58] applied multi-objective optimization for the design of a satelliteheat pipe. The problem concerned finding the heat pipe dimensions and operating temperature to minimize the heat pipe's mass while maximizing its thermal conductance.

\section{Unconstrained and constrained problems}

Omran and Mahdavi [7] compared the performance of the original HS, IHS $[82,83]$ and a new Global-best HS (GHS) algorithm on ten continuous functions of up dimension 100 , and six integer programming problems with up to 30 variables. They also studied the sensitivity to HS parameters and the effect of noise. Mukhopadhyay et al [94] compared another modified HS method with IHS, GHS and Differential Evolution (DE) for five continuous test functions. Gao et al [95] constructed a modified HS method purposely for multimodal functions and used it to three 2-dimensional multimodal functions. Optimization of a selection of continuous functions with 2-30 variables using the hybrid Harmony Annealing Algorithm (HAA) was reported in [96, 97]. The Rastrigin, Griewank and Sphere functions were optimized for 30, 50 and 100 variables using a hybrid PSO-HS algorithm developed for high-dimension problems in [98]. Results for the same three functions in 2-30 dimensions were reported in [100] for a GA-HS algorithm. A hybrid HS-DE method for uni-modal problems was tested on eight 50-dimensional benchmark functions in [95]. Jang et al [101] optimized two unconstrained and three constrained bench-mark functions with 2-7 continuous variables using a hybrid NM-HS method. A hybrid algorithm that combined elements from GA, HS, NM and the Tabu Search (TS) was tested on six continuous functions of dimension 2 to 10 in [99]. Fesanghary et al [74] applied a modified HS-SQP method to two constrained and two unconstrained benchmark problems.

Liu and Feng [85] used HS for system identification. They estimated ten discrete valued parameters of a Controlled Auto-Regressive Moving Average (CARMA) model for oil well heat wash data. Two applications in process control have been reported: nonlinear model predictive control for set-point tracking using HAA [86], and synchronization of discretetime chaotic systems using another modified HS method [87]. more applications include the solution of Sudoku puzzles [88], musical composition [89], timetabling and room allocation for university courses [25], optimization of a milling process [90], and the selection of land parcels for ecological conservation (a MCSP) [65]. Fesanghary et al [56] compared IHS and GA for the optimal design of a shell and tube heat exchanger, which included both annualized capital and operating costs. The optimal operation of a system of cogeneration (combined heat and power) plants was studied in [93]. The aim was to determine the heat and power generation rates at each plant to minimize the total cost, subject to constraints on the total heat and power demand, and the feasible operating region for each plant

\subsection{Applications in Computer Science}

The HS algorithm has been recently applied in many applications in computer science and engineering, among them: The clustering or grouping of web pages, the summary or text summarization, Internet routing and robotics.

Clustering is a problem of great practical importance that has been the focus of substantial research in several domains for decades. Given the dimensions and the properties of the documents, the clustering tends to be a much more difficult task when it comes to web documents. HS has been successfully applied to the problem of clustering of web pages, both for continuous data representation [34] as for discrete data representation [26]. HS based in clustering has been hybridized with the k-means algorithm HSCLUST resulting in [35]. Results show that the clustering of web documents based on HS is a good choice when it comes to partition large quantities of documents [24]. Another algorithm for Web document clustering is IGBHSK, this algorithm hybridized GHS and K-means. IGBHSK has the ability to define automatically the number of clusters for a problem given. This algorithm shows better results when used a matrix frequent terms document, framework of a vector model representation of documents, when the Bayesian information criterion is used as fitness function and when using the cosine similarity to compare documents [36].

Visual tracking is commonly used systems to correctly identify a target arbitrary in a video sequence. In this type of problems is consider the possibility that the target comes and 
disappear, change in size, is covered by another object in the video sequence, with the aim of quickly locate and efficiently. The most popular method used in visual tracking is the Kalman filter and the particle filter. HS and IHS have been used to create the Harmony Filter (HF) [25], which uses the Bhattacharyya coefficient to compare color histograms in the video sequence. HF gained further accuracy in the process of monitoring and recovery of target situations in which the other two algorithms did not Robotics is one of the sciences in which HS has been successfully applied over other evolutionary algorithms and other mathematical techniques. Calculating the coordinates of the movements of the robots is strong in this type of applications, among which are the calculation of optimal trajectories [37] and an application for a prototype reconfigurable mobile robot [38]. In [37] HS was used for minimizing the duration of motion, which is the main restriction of trajectories problems that have primarily aimed at increasing productivity, for robots used in the industrial field, in this case a robot manipulator with 6 degrees of freedom. HS was hybridized with the sequential quadratic programming (SQP, Sequential acronyms Quadratic Programming), resulting HHSA to improve the solutions calculated trajectories, using randomness in order to find the optimal initial values which are to be used for SQP vectors.

In [39], two new algorithms (HSPR and HSNPI) were proposed to solve the problem of lower cost with delay constraint on the bandwidth based on HS. HSPR and HSNPIwere evaluated in regard to their performance and efficiency against a GA and a modified version of BSMA. Simulation results of randomly generated networks and topologies indicatedthat the proposed algorithm exceeded HSNP.

\subsection{Applications in Electrical Engineering}

The method to detect the most efficient way, with the lower operating cost and reliably maintain one power system, within the constraints operational resources available generation is called Economic Dispatch (Economic Dispatch). Several mathematical programming methods have been employed to solve it, such as linear programming, nonlinear homogeneous programming [1, 40,41]. Considering the use of various types of fuels, in implementing functions can be found not converging unsmooth, prohibitive operative zones, between other, which makes such problems are not resolved by traditional mathematical programming models, and dynamic programming models and programming no mixed integer linear, although these methods tend to create an expansion of the dimensions of the problem [41]. HS has been used in this area as an alternative technique to the traditional methods due to its ability to scan to find high-performance regions within the space in a reasonable time. Its application is an alternative one of the most difficult problems Economic Dispatch that arises in Heat and Power Systems [40]. Within the design of electrical systems, it tries to find thebetter and safer operating point for each load situation [42]. In general, the design problems electrical systems are highly non-linear problem constraints and large scale. HS has been used to solve such type of electrical problems, in which a modified Search called
Harmonic with the Population Variance (PVHS) [43], where "control parameter known as bandwidth was matched to the standard deviation Current population "(free translation) [43]. The results proved its effectiveness after being subjected IEEE Test System 30.

In the field of photo-electronic detection, a version called Adaptive Harmony Search (AHS) has been used to solve the problem of Photon density wave within the witch "fits harmoniously memory throughout the process given search rate and adjusting HCMR tone (PAR) [44]. This method is used to locate abnormalities embedded in the sample according with location parameters to diagnose [44]. In optimizing multilevel inverters, there is a new HS method based on optimizing the waveform harmony step for such investors. The method proposed had the advantage of high rate of convergence and accuracy compared with other conventional methods of Optimization [45]. As a case study, the method was applied and tested on an investor of 13 levels. Simulation Results showed the effectiveness and flexibility of the proposed method [45].

In the field of mobile networks, a new guided optimization method for detecting randomly multiuser Direct-Sequence Code Division Multiple Access (DS-CDMA) was proposed [46]. HS was combined with the multiuser detection algorithm on a workbench channel decoding. Results obtained were close to those expected without using a search optimal detection multiuser systems even with high load [46].

\subsection{Applications in Civil Engineering}

Minimizing costs and space materials, are some of the most important features in the design, development and implementation of a structure or building. HS has undergone multiple related applications construction, network design and optimization of resources, excellent results compared to other algorithms in the minimizing the total cost of construction (production), that for civil engineering is the main factor and every company looks, leaving aside the quality of materials and construction itself. When speech structural design refers to the structure or frame construction, that is, the beams and columns steel, which support the weight of the construction [47-49].

In the design of hydraulic networks, the main purpose of using the HS is optimizing costs considering the dimensions of the pipes used in the network. HS was adapted to select the diameter for each segment of the pipeline in order to minimize the cost total network. To this,restrictions imposed by fluid mechanics were taken into account, i.e. mass equations and energy conservation, which is determined by the pressure minimum that must be submitted each section (segment pipe) $[3,4]$. To test the solutions in [4], a software package that allows evaluation of water quality distribution systems of drinking water (EPANET) was used [50].

\subsection{Applications in Economics}

A classic and important problem in economics is the sum radii. The sum of radii is part of the fractional programming, 
which aims at optimizing one or more radios of various functions. This problem considered very difficult because it involves nonconvex and/or multimodal functions. Among the applications of the problems sum of radii are: government contracting, the transportation science, finance, economics, engineering [2]. Furthermore, in their research part, these problems have theoretical and computational challenges.[63]. Proposed Harmony Search (PHS ), was proposed is another modification applied to handle such issues. In PHS, $B W$ was adjusted based on the central difference finite to approximate a derivative. The results Himmelblau optimizing function, show that the PHS obtained a better performance compared with initial HS, as the number of iterations. When applying the solution PHS various radii addition problems, did better results in the objective value over other methods.

\subsection{Applications in Ecology}

In modern life, industrial and urban conservation ecosystems and their species are very important. To achieve this, different techniques have been developed and used for the selection problem. In the field of optimization,five classes of reserve selection problems have been identified: species recovery problem (Species September Covering Problem, SSCP) recovery problem Maximum species (Maximal Covering Species Problem, MCSP), maximum representation problem of multiple species (Maximal Multiple-Species Representation Problem, MMRSP) problem restricting opportunity preservation (chance constrained covering problem) and problem preservation of expected (expected covering problem). Of the five proposed optimization problems, MCSP has been known as the most commonly used model $[51,65,66]$. HS was modified to adapt to various specific features of the problem such as: limited selection (sparse selection), selection of the first major book (big-bookfirst selection) and selection of the first diversity (diversityfirst selection). Although HS did not reach the global optimum for the problem with the MCSP model, it obtained many results close. This is important for the identification of alternative optimal solutions is valuable for planning and decision, as the case may be absent of a reservation [51].

\section{CONCLUSION AND FUTURE WORK}

Harmony search algorithm has proved to be a powerful tool for solving several optimization problems. It does not need any mathematical calculations to obtain the optimal solutions.

In recent years, HS was applied to many optimization problems, demonstrating its efficiency compared to other heuristic algorithms and other Meta mathematical optimization techniques. Continuous development improvements to the algorithm and various applications to new types of problems (operations research ,economy, computer science, civil engineering and electrical engineering), indicate that HS is a good choice. As a consequence, many studies have been proposed to increase its efficiency. However, there are many future works that can be done, such as:

1. Investigation of how to avoid being stuck in local solution, since most of the proposed HS had a problem of being, so.

2. Apply HS on dynamic problems.
3. Develop and analyze ensemble of HS operators algorithms.

4. Propose new adaptive mechanism to update HS parameters

5. Solve other real-world problem, such as travelling salesman problem and time series forecasting.

6. Apply HS to solve machine learning problems.

7. Investigate the performance of HS on solving constrained problems.

8. Analyze the effect of hybridizing HS with other EAs.

\section{REFERENCES}

[1]. Lee, K.S., Geem, Z.W., Lee, S.-H. and Bae, K.-W. The harmony search heuristic algorithm for discrete structural optimization", 2005, Engineering Optimization, 37(7): 663684.

[2] Yang, X.-S., Harmony Search as a Metaheuristic Algorithm, in Music-Inspired Harmony Search Algorithm. 2009, Springer Berlin / Heidelberg. p. 1-14.

[3] Geem, Z.W., Optimal Design of Water Distribution Networks Using Harmony Search. 2009: p. 112.

[4] Geem, Z.W., Optimal cost design of water distribution networks using harmony search. Engineering Optimization, 2006. 38.

[5] Geem, Z.W., Harmony search algorithms for structural design optimization, in Studies in computational intelligence, v.239. 2009, Springer Berlin Heidelberg: Berlin, Heidelberg. p. 228.

[6] Geem, Z.W., Harmony search algorithm for solving Sudoku, in Proceedings of the 11th international conference, KES 2007 and XVII Italian workshop on neural networks conference on Knowledge-based intelligent information and engineering systems: Part I. 2007, Springer-Verlag: Vietri sul Mare, Italy.

[7] Omran, M.G.H. and M. Mahdavi, Global-best harmony search. Applied Mathematics and Computation, 2008. 198(2): p. 643-656.

[8] Mahdavi, M., M. Fesanghary, and E. Damangir, An improved harmony search algorithm for solving optimization problems. Applied Mathematics and Computation, 2007. 188(2): p. 1567-1579.

[9] Zou, D., et al., A novel global harmony search algorithm for reliability problems. Computers \& Industrial Engineering, 2010. 58(2): p. 307-316.

[10] Geem, Z.W., et al., Recent Advances in Harmony Search. Advances in Evolutionary Algorithms, 2008: p. 16.

[11] Geem, Z., State-of-the-Art in the Structure of Harmony Search Algorithm, in Recent Advances In Harmony Search Algorithm. 2010, Springer Berlin / Heidelberg. p. 1-10.

[12] Geem, Z., J. Kim, and G.V. Loganathan, A New Heuristic Optimization Algorithm: Harmony Search. Simulation, 2001. 76(2): p. 60-68.

[13] Lee, K. and Z. Geem, A new meta-heuristic algorithm for continuous engineering optimization: harmony search theory and practice. Computer Methods in Applied Mechanics and Engineering, 2005. 194(36-38): p. $3902-$ 3933. 
[14] Geem, Z.W., Recent Advances In Harmony Search Algorithm. Studies in Computational Intelligence. Vol. 270. 2010, Annandale,Virginia: Springer

[15] Bäck, T., Evolutionary Algorithms in Theory and Practice. 1995: p. 328.

[16] Kirkpatrick, S., Optimization by simulated annealing: Quantitative studies. Journal of Statistical Physics, 1984. 34(5-6): p. 12.

[17] Enrique Alba, R.M., Tabu Search 2006. 36: p. 13.

[18] Glover, F., et al., The Ant Colony Optimization Metaheuristic: Algorithms, Applications, and Advances, in Handbook of Metaheuristics. 2003, Springer New York. p. 250-285.

[19] Vázquez, K., Ant Colony Optimization. Genetic Programming and Evolvable Machines, 2005. 6(4): p. 459460.

[20] Poli, R., J. Kennedy, and T. Blackwell, Particle swarm optimization. Swarm Intelligence, 2007. 1(1): p. 33-57.

[21] Mira, J., J. Álvarez, and X.-S. Yang, Engineering Optimizations via Nature-Inspired Virtual Bee Algorithms, in Artificial Intelligence and Knowledge Engineering Applications: A Bioinspired Approach. 2005, SpringerBerlin / Heidelberg. p. 317-323.

[22] Yang, X.-S., Nature-Inspired Metaheuristic Algorithms. 2008: p. 128.

[23] Watanabe, O., T. Zeugmann, and X.-S. Yang, Firefly Algorithms for Multimodal Optimization, in Stochastic Algorithms: Foundations and Applications. 2009, Springer Berlin / Heidelberg. p. 169-178.

[24] Forsati, R. and M. Mahdavi, Web Text Mining Using Harmony Search, in Recent Advances In Harmony Search Algorithm. 2010, Springer Berlin / Heidelberg. p. 51-64.

[25] Fourie, J., S. Mills, and R. Green, Visual Tracking Using Harmony Search, in Recent Advances In Harmony Search Algorithm. 2010, Springer Berlin / Heidelberg. p. 37-50.

[26] Mahdavi, M. and H. Abolhassani, Harmony K-means algorithm for document clustering. Data Mining and Knowledge Discovery, 2009. 18(3): p. 370-391.

[27] Al-Betar, M.A., A.T. Khader, and T.A. Gani, A harmony search for university course timetabling. 2008: p. 12.

[28] Mahdavi, M., Solving NP-Complete Problems by Harmony Search, in Music-Inspired Harmony Search Algorithm. 2009, Springer Berlin / Heidelberg. p. 53-70.

[29] Geem, Z.W. and K.-B. Sim, Parameter-setting-free harmony search algorithm. Applied Mathematics and Computation, 2010. 217(8): p. 3881-3889.

[30] Das, S., et al., Exploratory Power of the Harmony Search Algorithm: Analysis and Improvements for Global Numerical Optimization. Systems, Man, and Cybernetics, Part B: Cybernetics, IEEE Transactions on. 41(1): p. 89106.

[31] Geem, Z.W., Particle-swarm harmony search for water network design. Engineering Optimization, 2009. 41: p. 297-311.

[32] Geem, Z.W., Music-Inspired Harmony Search Algorithm: Theory and Applications. Studies in Computational Intelligence. Vol. 191. 2009, Rockville, Maryland: Springer Publishing Company, Incorporated. P.206.
[33] Geem, Z.W. and J.-Y. Choi, Music Composition Using Harmony Search Algorithm. Applications of Evolutionary Computing, 2007. 4448/2007: p. 7.

[34] Mahdavi, M., et al., Novel meta-heuristic algorithms for clustering web documents. Applied Mathematics and Computation, 2008. 201(1-2): p. 441-451.

[35] Forsati, R., et al. Hybridization of K-Means and Harmony Search Methods for Web Page Clustering. In Web Intelligence and Intelligent Agent Technology, 2008.WIIAT '08. IEEE/WIC/ACM International Conference on. 2008

[36] Cobos, C., et al. Web document clustering based on Global-Best Harmony Search, K-means, Frequent Term Sets and Bayesian Information Criterion. in IEEE Congress on Evolutionary Computation (IEEE CEC). 2010. Barcelona, Spain: IEEE.

[37] Tangpattanakul, P., A. Meesomboon, and P. Artrit, Optimal Trajectory of Robot Manipulator Using Harmony Search Algorithms, in Recent Advances In Harmony Search Algorithm. 2010, Springer Berlin / Heidelberg. p. 23-36.

[38] Xu, H., et al., Harmony Search Optimization Algorithm: Application to a Reconfigurable Mobile Robot Prototype, in Recent Advances In Harmony Search Algorithm. 2010, Springer Berlin / Heidelberg. p. 11-22.

[39] Forsati, R., A.T. Haghighat, and M. Mahdavi, Harmony search based algorithms for bandwidth-delay-constrained least-cost multicast routing. Computer Communications, 2008. 31(10): p. 2505-2519.

[40] Fesanghary, M., An Introduction to the Hybrid HSSQP Method and Its Applications, in Recent Advances In Harmony Search Algorithm. 2010, Springer Berlin / Heidelberg. p. 99-109.

[41] Fesanghary, M., Harmony Search Applications in Mechanical, Chemical and Electrical Engineering, in MusicInspired Harmony Search Algorithm. 2009, Springer Berlin/ Heidelberg. p. 71-86.

[42] Geem, Z.W., Recent Advances in Harmony Search Algorithm. 2010: p. 176.

[43] Panigrahi, B., et al., Population Variance Harmony Search Algorithm to Solve Optimal Power Flow with NonSmooth Cost Function, in Recent Advances In Harmony Search Algorithm. 2010, Springer Berlin / Heidelberg. p. 65-75.

[44] Dong, H., et al. Improved harmony search for detection with photon density wave. in International Symposium on Photoelectronic Detection and Imaging 2007: Related Technologies and Applications. 2007. Beijing, China: SPIE

[45] Majidi, B., et al. Harmonic optimization in multi-level inverters using harmony search algorithm. in Power and Energy Conference, 2008. PECon 2008. IEEE 2ndInternational. 2008.

[46] Rong, Z. and L. Hanzo, Iterative Multiuser Detection and Channel Decoding for DS-CDMA Using Harmony Search. Signal Processing Letters, IEEE, 2009. 16(10): p. 917-920.

[47] Saka, M.P., Optimum design of steel sway frames to BS5950 using harmony search algorithm. Journal of Constructional Steel Research, 2009. 65(1): p. 36-43.

[48] Geem, Z. and S. Degertekin, Optimum Design of Steel Frames via Harmony Search Algorithm, in Harmony Search Algorithms for Structural Design Optimization. 2009, Springer Berlin / Heidelberg. p. 51-78. 
[49] Saka, M. and F. Erdal, Harmony search based algorithm for the optimum design of grillage systems to LRFD-AISC. Structural and Multidisciplinary Optimization, 2009. 38(1):p. 25-41.

[50] Rossman, L.A., EPANET- Users Manual. 1994: p. 7.

[51] Geem, Z., C.-L. Tseng, and J. Williams, Harmony Search Algorithms for Water and Environmental Systems, in Music-Inspired Harmony Search Algorithm. 2009, Springer Berlin / Heidelberg. p. 113-127.

[52] Kim, J.H., Z.W. Geem, and E.S. Kim, Parameter Estimation Of The Nonlinear Muskingum Model Using Harmony Search1. JAWRA Journal of the American Water Resources Association, 2001. 37(5): p. 1131-1138.

[53] Ayvaz, M., Identification of Groundwater Parameter Structure Using Harmony Search Algorithm, in MusicInspired Harmony Search Algorithm. 2009, Springer Berlin/ Heidelberg. p. 129-140.

[54] Ayvaz, M.T., Simultaneous determination of aquifer parameters and zone structures with fuzzy c-means clustering and meta-heuristic harmony search algorithm.Advances in Water Resources, 2007. 30(11): p. 2326-2338.

[55] Cheng, Y.M., et al., An improved harmony search minimization algorithm using different slip surface generation methods for slope stability analysis. EngineeringOptimization, 2008. 40(2): p. 95 - 115.

[56] Fesanghary, M., E. Damangir, and I. Soleimani, Design optimization of shell and tube heat exchangers using global sensitivity analysis and harmony search algorithm. Applied Thermal Engineering, 2009. 29(5-6): p. 1026-1031.

[57] Khorasany, R.M. and M. Fesanghary, A novel approach for synthesis of cost-optimal heat exchanger networks. Computers \& Chemical Engineering, 2009. 33(8): p. 13631370 .

[58] Geem, Z.W. and H. Hwangbo, Application of Harmony Search to Multi-Objective Optimization for Satellite Heat Pipe Design. 2006

[59] Sam Ryu, A.S.D., Caspar N. Heyl, Zong Woo Geem, Mooring Cost Optimization Via Harmony Search.Proceedings of OMAE07 - 26thh International Conference on Offshore Mechanics and Arctic Engineering, 2007: p. 8

[60] Mohsen, A., A. Khader, and D. Ramachandram, An Optimization Algorithm Based on Harmony Search for RNA Secondary Structure Prediction, in Recent Advances In Harmony Search Algorithm. 2010, Springer Berlin / Heidelberg. p. 163-174.

[61] Alexandre, E., L. Cuadra, and R. Gil-Pita, Sound Classification in Hearing Aids by the Harmony Search Algorithm, in Music-Inspired Harmony Search Algorithm. 2009, Springer Berlin / Heidelberg. p. 173-188.

[62] Panchal, A., Harmony Search in Therapeutic Medical Physics, in Music-Inspired Harmony Search Algorithm. 2009, Springer Berlin / Heidelberg. p. 189-203.

[63] Jaberipour, M. and E. Khorram, Solving the sum-ofratios problems by a harmony search algorithm. Journal of Computational and Applied Mathematics, 2010. 234(3):p. 733-742.

[64] Geem, Z.W., Application of Harmony Search to Vehicle Routing. American Journal of Applied Sciences, 2005: p. 6.
[65] Geem, Z.W., Williams, J.C., Harmony search and ecological optimization. International Journal of Energy and Environment 1, 2007: p. 150 - 154.

[66] Geem, Z.W. and J.C. Williams, Ecological optimization using harmony search, in Proceedings of the American Conference on Applied Mathematics. 2008, WorldScientific and Engineering Academy and Society (WSEAS): Cambridge, Massachusetts.

[67] Alatas, B. Chaotic harmony search algorithms, Applied Mathematics andComputation, 2010 p. $2687-$ 2699.

[68] N. Taherinejad, Highly reliable harmony search algorithm, Eur. Conf. Circuit Theory Des. (2009) 818-822.

[69] Z.W. Geem, W.E. Roper, Various continuous harmony search algorithms for web-based hydrologic parameter optimization, Int. J. Math. Model. Numer.Optim. 1 (3) (2010) 213-226

[70] Ryu, S., Duggal, A.S., Caspar, N.H., et al.: Mooring cost optimization via harmony search.In: 26th International Conference on Offshore Mechanics and Arctic Engineering (OMAE2007), San Diego, USA, June 10-15 (2007).

[71]. Mahdavi, M., Fesanghary, M., Damangir, E.: An improved harmony search algorithm forsolving optimization problems. Applied Mathematics and Computation 188, 1567-1579(2007)

[72] Geem, Z.W., Tseng, C.-L., Park, Y.-J.: Harmony search for generalized orienteering problem:Best touring in china. In: Wang, L., Chen, K., S. Ong, Y. (eds.) ICNC 2005. LNCS, vol. 3612, pp. 741-750. Springer, Heidelberg (2005)

[73] Ceylan, H., Ceylan, H., Haldenbilen, S., et al.: Transport energy modeling with metaheuristicharmony search algorithm, an application to Turkey. Energy Policy 36, 2527-2535 (2008)

[74] Geem, Z.W.: School bus routing using harmony search. In: Genetic and EvolutionaryComputation Conference (GECCO 2005), Washington DC, USA, June 25-29 (2005)

[75] Liu, T.-N., Feng, Z.-B.: Adaptive identification and filtering based on harmony search.Journal of Jilin University (Information Science Edition) 22, p. 306-309 (2004) (in Chinese)

[76] Tian, Y.-H., Bo, Y.-M., Gao, M.-F.: The application of harmony annealing algorithm forpredictive control of nonlinear systems. Automation in Petro-Chemical Industry 2, p. 39-42(2005) (in Chinese)

[77] Santos Coelho, L.d., de Andrade Bernert, D.L.: An improved harmony search algorithmfor synchronization of discrete-time chaotic systems. Chaos, Solitons \& Fractals (2008),doi:10.1016/j.chaos.2008.09.028

[78] Geem, Z.W.: Harmony search algorithm for solving sudoku. In: Apolloni, B., Howlett,R.J., Jain, L. (eds.) KES 2007, Part I. LNCS (LNAI), vol. 4692, p. 371-378. Springer,Heidelberg (2007)

[79] Geem, Z.W., Choi, J.-Y.: Music composition using harmony search algorithm. In: Giacobini,M. (ed.) EvoWorkshops 2007. LNCS, vol. 4448, pp. 593-600. Springer, Heidelberg(2007)

[80] Zarei, O., Fesanghary, M., Farshi, B., et al. Optimization of multi-pass face-milling via harmonysearch algorithm. Journal of Materials Processing Technology (2008), doi:10.1016/j.jmatprotec.2008.05.029 
[81] Vasebi, A., Fesanghary, M., Bathaee, S.M.T.: Combined heat and power economic dispatchby harmony search algorithm. International Journal of Electrical Power \& EnergySystems 29, p. 713-719 (2007)

[82] Mukhopadhyay, A., Roy, A., Das, S., et al.: Populationvariance and explorative power ofharmony search: an analysis. In: Second National Conference on Mathematical Techniques:Emerging Paradigms for Electronics and IT Industries (MATEIT 2008), NewDelhi, India, September 26-28 (2008)

[83] Gao, X.Z., Wang, X., Ovaska, S.J.: Modified harmony search methods for uni-modal andmulti-modal optimization. In: 8th International Conference on Hybrid Intelligent Systems(HIS 2008), Barcelona, Spain, September 10-12 (2008)

[84] Tian, Y.-H., Bo, Y.-M., Gao, M.-F.: Harmony annealing algorithm for multi-dimensionalfunction optimization. Computer Simulation 21, 79-82 (2004) (in Chinese)
[85]Tian, Y.-H., Bo, Y.-M., Gao, M.-F.: Parameters choice criteria in harmony annealing forfunction optimization. Computer Simulation 22, 70-74+89 (2005) (in Chinese)

[86] Li, H.-Q., Li, L.: A novel hybrid particle swarm optimization algorithm combined withharmony search for high dimensional optimization problems. In: 2007 International Conferenceon Intelligent Pervasive Computing (IPC 2007), Jeju Island, Korea, October 11-13(2007)

[87] Li, Q., Yang, S., Ruan, Y.: A hybrid algorithm for optimizing multi-modal functions. WuhanUniversity Journal of Natural Sciences 11, 551-554 (2006)

[88] Li, H.-Q., Li, L.: A novel hybrid real-valued genetic algorithm for optimization problems.In: 2007 International Conference on Computational Intelligence and Security (CIS 2007),Harbin, China, December 15-19 (2007)

[89] Jang, W.S., Kang, H.I., Lee, B.H.: Hybrid simplexharmony search method for optimizationproblems. In: 2008 IEEE Congress on Evolutionary Computation, HongKong, China, June 1-6 (2008) 